\title{
Prevalencia de consumo de alcohol en estudiantes universitarios
}

Prevalence of alcohol consumption in university students

\author{
María Esther Barradas Alarcón \\ Universidad Veracruzana,México \\ maeba107@hotmail.com \\ Nohemí Fernández Mojica \\ Universidad Veracruzana,México \\ nfernandez@uv.mx \\ Leticia Gutiérrez Serrano \\ Universidad Veracruzana,México \\ legutierrez@prodigy.net.mx
}

\section{Resumen}

El objetivo del presente trabajo es estimar la prevalencia del consumo de alcohol en los alumnos de la Universidad Veracruzana, Campus Veracruz, México. Para ello se utilizó el enfoque cuantitativo y la investigación descriptiva de corte transversal no experimental (ex post facto). El instrumento aplicado fue el Cuestionario sobre el Consumo de Drogas en Estudiantes Universitarios (CODEU, 2012), disponible en la página oficial de la UV por tres semanas. De una muestra de 4821 estudiantes voluntarios, 1 911(39.6\%) fueron hombres y 2910 (60.4\%) mujeres, todos ellos estudiantes de carreras en las áreas de Artes, Biológico-Agropecuaria, Ciencias de la Salud, Económico-Administrativa, Humanidades y Técnica. El análisis de la información se realizó con base en estadística descriptiva utilizando software IBM SPSS Statistic versión 20. En su mayoría (83 \%), los estudiantes mencionaron que alguna vez en su vida habían tomado una bebida completa. En cuanto a su nivel de consumo, $7.8 \%$ mencionó tener un consumo de riesgo, $17.4 \%$ señaló tener un 
consumo abusivo, mientras que $52.8 \%$ dijo tener un consumo moderado. El área académica de mayor prevalencia en el consumo de alcohol riesgoso fue Artes con $15.7 \%$, mientras que la mayor prevalencia de consumo abusivo recayó en el área de Ciencias biológicas agropecuarias con $29.1 \%$, sin embargo, en general se reconoció que todas las áreas presentaron porcentajes mayores al consumo moderado. Los resultados obtenidos por este estudio muestran claramente la presencia de porcentajes altos de consumo de alcohol en los estudiantes universitarios, lo que indica la urgencia de planear y poner en marcha estrategias que traten y prevengan dicho consumo.

Palabras clave: prevalencia, consumo, alcohol, estudiantes.

\section{Abstract}

To estimate prevalence of alcohol consumption in students of Universidad Veracruzana, campus Veracruz, Mexico. A quantitative methodology was considered for this work, in a descriptive non-experimental cross-cutting (ex post facto) study. For this, a three week online Drug Consumption test was applied to students from the six different campus of Universidad Veracruzana (CODEU, 2012). In this article only the results of Veracruz-Boca del Rio campus are presented, in which 4,821 students participated: male $n=1911(39.6 \%)$ and female $n=2910$ (60.4\%), among the different majors in academic areas such as Arts, Biological, Agriculture and Livestock Sciences, Health Sciences, Economics and Management sciences, Humanistic and Technical Studies. The analysis of the information is based on Descriptive Statistics using IBM SPSS Statistic software 20. The $83 \%$ of the students mentioned to have had a complete drink once in their lives; from this $7.8 \%$ mentioned to have had a risky consumption level, $17.4 \%$ mentioned to have had abusive consumption, while $52.8 \%$ considered having a moderated consumption level. The Academic field with the highest prevalence in risky alcohol consumption was Arts with $15.7 \%$, while Agriculture and Livestock Sciences showed $29.1 \%$ of alcohol abusive consumption; in general, the highest percentages in moderated consumption were found in all the academic areas. The results obtained in this study clearly show high percentages of 
alcohol consumption in University students, which points out the importance of setting up treatment and prevention strategies in alcohol consumption.

Key words: prevalence, consumption, alcohol, students.

Fecha Recepción: $\quad$ Mayo 2015 Fecha Aceptación: Octubre 2015

\section{Introducción}

Actualmente en nuestra sociedad el consumo de alcohol forma parte de la vida cotidiana. Según muchos adultos y jóvenes no puede faltar el alcohol en las fiestas y reuniones. Además, sin importar la clase social a la que se pertenezca, ni la edad o la educación que se tengan, se suele creer que el consumo de alcohol ayuda a aliviar la tristeza, a infundir valor, a atenuar el temor o provocar alegría. La mayoría de los países reportan un alto índice de consumo, no solo en adultos y jóvenes sino también en adolescentes (Organización Mundial de la Salud). El Informe Mundial de Situación sobre Alcohol y Salud 2014, señala que cada año mueren en el mundo 3.3 millones de personas como consecuencia del consumo nocivo de alcohol ${ }^{1}$, lo cual representa al $5.9 \%$ de todas las defunciones. Asimismo, informó que el uso nocivo de alcohol es el factor causal de más de 200 enfermedades y trastornos. En general, mencionó que $5.1 \%$ de la carga mundial de morbilidad y lesiones es atribuible al consumo de alcohol, calculado en términos de la esperanza de vida ajustada en función de la discapacidad (EVAD). Asimismo, encontró que

el consumo de alcohol provoca defunción y discapacidad a una edad relativamente temprana. En el grupo etario de 20 a 39 años, $25 \%$ de las defunciones se atribuyen al consumo de alcohol. Existe una relación causal entre el consumo nocivo de alcohol y una serie de trastornos mentales y comportamentales, además de las enfermedades no transmisibles y los traumatismos. Recientemente se han determinado relaciones causales entre el consumo nocivo y la incidencia de enfermedades infecciosas tales como la 
tuberculosis y el VIH/SIDA. Finalmente, más allá de las consecuencias sanitarias, el consumo nocivo de alcohol provoca pérdidas sociales y económicas importantes, tanto para las personas como para la sociedad en su conjunto.

En México, el consumo de alcohol se ha considerado como la principal adicción que afecta especialmente a los adolescentes (Encuesta Nacional de Adicciones, ENA 2011). Además, la prevalencia de consumo en adolescentes de 12-17 años fue de 46-9\% en hombres y de $37.7 \%$ en mujeres, quienes han consumido alcohol alguna vez en su vida. En el último año, la prevalencia de consumo fue de $30.0 \%$ (31.8\% en hombres y $28.1 \%$ en mujeres). Los adolescentes presentaron $4.1 \%$ de dependencia al alcohol (6.2\% hombres y $2 \%$ mujeres). Por su parte, en la población adulta de 18-65 años la prevalencia de consumo de alcohol fue de $77.1 \%$; quienes han consumido alcohol alguna vez en su vida corresponde al $88.1 \%$ en los hombres y al $67.0 \%$ en las mujeres. En el último año, la prevalencia de consumo fue de $55.7 \%$ (69.3\% en hombres y $43.2 \%$ en mujeres). Asimismo, $6.6 \%$ de los adultos presentaron dependencia al alcohol (11.8\% en hombres y $1.7 \%$ en mujeres). Su consumo y complicaciones en los estudiantes de secundaria han sido identificados por el gobierno federal como un problema de salud pública mayor y como el principal problema de salud que aqueja a cualquier institución educativa. Se estima que la ingesta de bebidas alcohólicas en las instituciones desde el nivel secundaria hasta el superior tiene que ver anualmente con la muerte de 1400 estudiantes, 500000 lesiones, 600000 agresiones físicas y 70000 agresiones sexuales.

El alcohol es una droga de inicio para el consumo de otras drogas ilícitas como la cocaína y la marihuana. De acuerdo al Sistema de Vigilancia Epidemiológica de las Adicciones para Veracruz, esta fue la droga de inicio en 56.9.2 \% de los jóvenes, quienes después comenzaron a consumir una segunda sustancia. Además, para este mismo estado de la república el alcohol es la droga de mayor impacto con 55.2 \% (SISVEA, 2012). Entre las consecuencias que el alcohol genera están dificultades frecuentes con la familia, peleas, problemas con la policía, problemas laborales, pérdida del empleo, violencia y comisión de delitos. Un alto porcentaje de los menores de edad que son detenidos en el Consejo Tutelar de Menores por cometer delitos (43.3\%), se encontraban bajos los efectos del alcohol, siendo este la segunda droga más utilizada por ellos (SISVEA, 2012). Además, 8 de cada 100 lesionados por causa externa se encontraban alcoholizados al momento de ingresar al 
servicio de urgencias hospitalarias (SISVEA, 2009), y 6 de cada 100 personas mueren al año por cirrosis de hígado (la cuarta causa de mortalidad en general). Asimismo, es la principal causa de muerte en jóvenes de 15 a 29 años por accidentes de automotores (incluyendo atropellados). Se estima que casi la quinta parte de los accidentes laborales se relaciona con la ingesta de bebidas alcohólicas y que se encuentra entre las 10 principales causas de discapacidad de los trabajadores, afectando directamente su productividad y, por lo tanto, a la empresa.

En Veracruz un estudio realizado con estudiantes de la carrera de ingeniería industrial del Instituto Tecnológico de Veracruz, encontró que de 356 estudiantes, $84 \%$ consumen alcohol y $16 \%$ refirieron no consumirlo (Barradas 2009). Otro estudio realizado en esta misma institución solo que en las carreras de ingeniería química y licenciatura en administración se encontró que en la primera el porcentaje de alumnos hombres que consumen alcohol fue de $\mathrm{n}=8(88.9 \%)$ y de $\mathrm{n}=29(96 \%)$ en las mujeres; en la segunda carrera consumen alcohol $n=22(100 \%)$ hombres y $n=47(90.4 \%)$ mujeres (De los Santos F., Barradas A., Fernández M., Sánchez, B., 2010). La Universidad Veracruzana como institución de educación superior con sede en diferentes regiones del estado de Veracruz se ha ocupado por establecer programas tanto preventivos como de atención a los estudiantes. Estas acciones recayeron originalmente en el CENATI (Centro de Atención Integral para la Salud del Estudiante Universitario), y actualmente en el CENDIHU (Centro para el Desarrollo Humano e Integral de los Universitarios). Entre otras acciones cuenta con una Red Veracruzana de Investigación en Adicciones (REVIVA), constituida por 11 cuerpos académicos distribuidos en las cinco regiones académicas y por tres grupos de investigación ubicados en la Benemérita Universidad Autónoma de Puebla y en las universidades de San Buenaventura y Boyacá, Colombia. Asimismo, realizó una investigación con el objetivo general de conocer la prevalencia del consumo de drogas legales e ilegales, los factores y la percepción de riesgo en estudiantes de la Universidad Veracruzana para proponer estrategias preventivas basadas en evidencias. Estuvo dirigido a los 57286 estudiantes de licenciatura y de las carreras de Técnico Superior Universitario (TSU) inscritos en el periodo agosto 2012 - enero 2013, tanto en el sistema abierto como en el escolarizado y en la Universidad Veracruzana Intercultural (UV) en las cinco regiones y en todas las facultades, carreras y periodos, según datos proporcionados por la Dirección 
General de Administración Escolar, donde 20644 estudiantes contestaron el instrumento y de estos 4821 eran exclusivamente de la región Veracruz-Boca del Río.

\section{Método}

Tipo de investigación. El estudio tuvo un carácter descriptivo y su propósito fue determinar la incidencia y prevalencia del consumo de alcohol en alumnos de la Universidad Veracruzana, región Veracruz.

Método. Se utilizó la metodología cuantitativa, la cual Hernández, Fernández y Baptista (2014) es el método que utiliza la recolección y análisis de datos para contestar preguntas de investigación, confiando en la medición numérica, el conteo y frecuentemente en el uso de la estadística para establecer con exactitud patrones de comportamiento en una población.

Definición de la variable. Prevalencia en el consumo de alcohol.

Definición conceptual. Prevalencia alguna vez en la vida (índice que considera a la población que reportó haber tomado por lo menos una copa completa de alcohol alguna vez en la vida). Prevalencia último año (índice que considera a la población que reportó haber tomado por lo menos una copa completa de alcohol el año previo a la entrevista). Prevalencia último mes (índice que considera a la población que reportó haber tomado por lo menos una copa completa de alcohol los treinta días previos a la entrevista).

Instrumento. El instrumento utilizado para la realización del diagnóstico de consumo de drogas en estudiantes de la Universidad Veracruzana fue denominado Cuestionario sobre el Consumo de Drogas en Estudiantes Universitarios (CODEU, 2012) y es producto de una adaptación realizada con la autorización expresa de los autores del instrumento denominado Cuestionario de Estudiantes UNAM 2007, diseñado por Villatoro et al. (2008), aplicado con anterioridad en esa casa de estudios. El instrumento consta de 73 preguntas que indagan acerca de los siguientes aspectos: sociodemográficos, consumo de drogas lícitas e ilícitas, factores y percepción de los estudiantes sobre las políticas institucionales acerca del tema y campañas preventivas dirigidas a este grupo de edad por diversas instituciones con un índice de confiabilidad .787 en Alpha de Cronbach. 


\section{Resultados}

Los estudiantes que participaron en este estudio fueron 4 821, de los cuales $39.6 \%$ $(n=1911)$ eran hombres y $60.4 \%(n=2910)$ mujeres.

Dentro de las características sociodemográficas de los estudiantes destaca que la población fue mayoritariamente femenina $60.4 \%(n=2910)$, con una media de edad de 20.6 años. En cuanto al estado civil y los hijos, $\mathrm{n}=2364$ (49 \%) dijo ser soltero y $\mathrm{n}=4578$ (95\%) no tener hijos. En este sentido destacó el área académica Ciencias de la salud (tabla 2) con n=1743 (36.2\%). Con respecto a la generación, $\mathrm{n}=1897$ (39.3\%) están a la mitad de la carrera, $\mathrm{n}=1519$ (31.5), y al sistema educativo, $\mathrm{n}=4592$ (95.2\%) es escolarizado (tabla 1). 


\begin{tabular}{|c|c|c|c|}
\hline CATEGORÍA & CARACTERÍSTICA & $\mathbf{N}$ & $\%$ \\
\hline \multicolumn{4}{|l|}{ Sexo } \\
\hline & Hombre & 1911 & 39.6 \\
\hline & Mujer & 2910 & 60.4 \\
\hline \multicolumn{4}{|l|}{ Edad (media) } \\
\hline & 20.6 años de edad & & \\
\hline \multicolumn{4}{|l|}{ Estado civil } \\
\hline & Soltero & 2364 & 49.0 \\
\hline & Casado & 73 & 1.5 \\
\hline & Vivo en unión libre & 112 & 2.3 \\
\hline & Divorciado & 26 & .5 \\
\hline \multicolumn{4}{|l|}{ Hijos } \\
\hline & Sí & 243 & 5.0 \\
\hline & No & 4578 & 95.0 \\
\hline \multicolumn{4}{|l|}{ Número de hijos } \\
\hline & 1 & 187 & 3.8 \\
\hline & 2 & 42 & .9 \\
\hline & 3 & 11 & .2 \\
\hline & 4 & 3 & .1 \\
\hline \multicolumn{4}{|l|}{ Área académica } \\
\hline & Artes & 19 & .4 \\
\hline & $\begin{array}{c}\text { Ciencias Biológicas } \\
\text { Agropecuarias }\end{array}$ & 487 & 10.1 \\
\hline & Ciencias de la Salud & 1743 & 36.2 \\
\hline & $\begin{array}{l}\text { Económico- } \\
\text { Administrativa }\end{array}$ & 1309 & 27.2 \\
\hline & Humanidades & 877 & 18.2 \\
\hline & Técnica & 386 & 8.0 \\
\hline \multicolumn{4}{|l|}{ Generación } \\
\hline & Iníciales & 1519 & 31.5 \\
\hline & Intermedios & 1897 & 39.3 \\
\hline & Avanzados & 1040 & 21.6 \\
\hline & Rezagados & 77 & 1.6 \\
\hline & No especificado & 288 & 6.0 \\
\hline \multicolumn{4}{|l|}{ Sistema } \\
\hline & Escolarizado & 4592 & 95.2 \\
\hline & Abierto & 229 & 4.8 \\
\hline
\end{tabular}

Tabla 1. Características sociodemográficas de los estudiantes participantes. Fuente: Consumo de Drogas en Estudiantes Universitarios (CODEU, 2012). 
En el área de Artes, $94.74 \%$ de los estudiantes han consumido en cantidad al menos una bebida alcohólica completa en su vida (copa, caballito, lata...), por ejemplo, cerveza, tequila, vino, ron, cooler, brandy, vodka, cuba, piña colada, medias de seda, etcétera (tabla 2).

\begin{tabular}{|l|c|c|c|c|}
\hline \multirow{2}{*}{ Área académica } & \multicolumn{3}{|c|}{ Sí } & \multicolumn{3}{c|}{$\mathrm{NO}$} \\
\cline { 2 - 5 } & $f$ & $\%$ & $f$ & $\%$ \\
\hline Artes $(\mathrm{n}=19)$ & 18 & 94.74 & 1 & 5.26 \\
\hline Biológico-Agropecuaria $(\mathrm{n}=487)$ & 431 & 88.501 & 56 & 11.499 \\
\hline Ciencias de la Salud $(\mathrm{n}=1743)$ & 1450 & 83.19 & 293 & 16.81 \\
\hline Económico-Administrativa ( $\mathrm{n}=1309)$ & 1067 & 81.51 & 242 & 18.49 \\
\hline Humanidades $(\mathrm{n}=877)$ & 744 & 84.83 & 133 & 15.17 \\
\hline Técnica $(\mathrm{n}=386)$ & 295 & 76,4 & 91 & 23,5 \\
\hline Total & 4005 & 83 & 815 & 17 \\
\hline
\end{tabular}

Tabla 2. Área de los estudiantes que al menos una vez en su vida han consumido una bebida completa. Fuente: Consumo de Drogas en Estudiantes Universitarios (CODEU, 2012).

El consumo de alcohol en los jóvenes universitarios inició en promedio a los 16 años de edad, lo cual resulta bastante interesante puesto que una gran cantidad de ellos probaron las bebidas alcohólicas cuando todavía eran menores de edad y les estaba prohibido (tabla 3).

Tabla 3 Prevalencia del consumo de alcohol

\begin{tabular}{|c|c|c|c|}
\hline Variable & Características & $F x$ & $\%$ \\
\hline \multirow{2}{*}{$\begin{array}{l}\text { Alguna vez en tu vida has tomado } \\
\text { una bebida completa }\end{array}$} & Sí & 4 & 83.1 \\
\hline & $\mathrm{No}$ & 816 & 16.9 \\
\hline $\begin{array}{l}\text { Edad promedio de inicio de } \\
\text { consumo }\end{array}$ & 16 años & & \\
\hline \multirow{7}{*}{ Tipo de bebida de mayor consumo } & Vino & 935 & 19.4 \\
\hline & Coolers & 539 & 11.2 \\
\hline & Cerveza & 2577 & 53.5 \\
\hline & Brandy, vodka, tequila, ron, whisky & 2440 & 50.6 \\
\hline & Cockteles (piña colada, medias de seda) & 1390 & 28.8 \\
\hline & Pulque & 89 & 1.8 \\
\hline & $\begin{array}{l}\text { Alcohol puro o aguardiente, solo o } \\
\text { preparado }\end{array}$ & 135 & 2.8 \\
\hline
\end{tabular}

Fuente: Consumo de Drogas en Estudiantes Universitarios (CODEU, 2012). 
La frecuencia del consumo de alcohol es de 1 a 3 veces al mes y la cantidad ingerida es de 1 a 4 copas por ocasión (20.1\%); seguida de un uso de 2 a 5 veces al año, y de 1 a 4 copas por ocasión $(19.0 \%)$, lo que hace evidente que los estudiantes universitarios consumen alcohol pero de manera moderada; sin embargo, $32.9 \%$ manifestó haber llegado a la embriaguez por lo menos una ocasión en el último año (tabla 4).

\section{Tabla 4. Prevalencia de consumo de alcohol}

\begin{tabular}{|c|c|c|c|}
\hline Variable & Características & $F x$ & $\%$ \\
\hline \multirow{2}{*}{$\begin{array}{l}\text { Cantidad y frecuencia de consumo de alcohol } \\
\text { (diario) }\end{array}$} & 1 a 4 copas por ocasión & 277 & 5.7 \\
\hline & 5 copas o más por ocasión & 17 & 4 \\
\hline \multirow{2}{*}{$\begin{array}{l}\text { Cantidad y frecuencia de consumo de alcohol } \\
\text { ( } 3 \text { a } 6 \text { veces por semana) }\end{array}$} & 1 a 4 copas por ocasión & 303 & 6.3 \\
\hline & 5 copas o más por ocasión & 40 & 0.8 \\
\hline \multirow{2}{*}{$\begin{array}{l}\text { Cantidad y frecuencia de consumo de alcohol } \\
\text { (1 a } 2 \text { veces por semana) }\end{array}$} & 1 a 4 copas por ocasión & 480 & 10 \\
\hline & 5 copas o más por ocasión & 225 & 4.7 \\
\hline \multirow{2}{*}{$\begin{array}{l}\text { Cantidad y frecuencia de consumo de alcohol } \\
\text { (1 a } 3 \text { veces por mes) }\end{array}$} & 1 a 4 copas por ocasión & 969 & 20.1 \\
\hline & 5 copas o más por ocasión & 471 & 9.8 \\
\hline \multirow{2}{*}{$\begin{array}{l}\text { Cantidad y frecuencia de consumo de alcohol } \\
\text { (6 a } 11 \text { veces al año) }\end{array}$} & 1 a 4 copas por ocasión & 674 & 14 \\
\hline & 5 copas o más por ocasión & 329 & 6.8 \\
\hline \multirow{2}{*}{$\begin{array}{l}\text { Cantidad y frecuencia de consumo de alcohol } \\
\text { ( } 2 \text { a } 5 \text { veces al año) }\end{array}$} & 1 a 4 copas por ocasión & 915 & 19 \\
\hline & 5 copas o más por ocasión & 283 & 5.9 \\
\hline \multirow{2}{*}{ Frecuencia y consumo ( 1 vez al año) } & 1 a 4 copas por ocasión & 790 & 16.4 \\
\hline & 5 copas o más por ocasión & 247 & 5.1 \\
\hline \multirow{3}{*}{$\begin{array}{l}\text { Frecuencia y consumo los fines de } \\
\text { semana }\end{array}$} & Siempre & 63 & 1.3 \\
\hline & Frecuentemente & 361 & 7.5 \\
\hline & Algunas veces & 2379 & 49.3 \\
\hline \multirow{5}{*}{ Frecuencia de embriaguez } & Nunca en el último año & 1663 & 34.5 \\
\hline & $\begin{array}{c}\text { Por lo menos } 1 \text { vez en el último } \\
\text { año }\end{array}$ & 1289 & 26.7 \\
\hline & Una vez en el último mes & 540 & 11.2 \\
\hline & $\begin{array}{c}\text { De dos a tres veces en el último } \\
\text { mes }\end{array}$ & 220 & 4.6 \\
\hline & $\begin{array}{l}\text { Una o más veces en la última } \\
\text { semana }\end{array}$ & 44 & 0.9 \\
\hline
\end{tabular}

Fuente: Consumo de Drogas en Estudiantes Universitarios (CODEU, 2012).

Cabe destacar que en todas las áreas alrededor del $50 \%$ reportó un nivel de consumo moderado seguido del consumo abusivo, destacando aquí el área de Artes (21 \%). Aunque el nivel de consumo de riesgo obtuvo porcentajes menores en todas las áreas, 
no deja de ser preocupante si se toma en cuenta que la población estudiada se caracteriza por estar en su mayoría a mitad de la carrera y por haber comenzado a consumir alcohol a partir de los 16 años de edad (tabla 5).

Tabla 5. Área académica y su nivel de consumo de alcohol

\begin{tabular}{|c|c|c|c|c|c|c|c|c|c|c|}
\hline \multirow[t]{2}{*}{ Área académica } & \multicolumn{2}{|c|}{$\begin{array}{c}\text { Consumo de } \\
\text { riesgo }\end{array}$} & \multicolumn{2}{|c|}{$\begin{array}{c}\text { Consumo } \\
\text { abusivo }\end{array}$} & \multicolumn{2}{|c|}{$\begin{array}{l}\text { Consumo } \\
\text { moderado }\end{array}$} & \multicolumn{2}{|c|}{$\begin{array}{c}\text { No } \\
\text { especificado }\end{array}$} & \multicolumn{2}{|c|}{ Total } \\
\hline & $f$ & $\%$ & $f$ & $\%$ & $f$ & $\%$ & $f$ & $\%$ & $f$ & $\%$ \\
\hline Artes $(n=19)$ & 3 & 15.79 & 4 & 21.05 & 10 & 52.63 & 1 & 5.26 & 18 & 94.73 \\
\hline $\begin{array}{l}\text { Biológico- } \\
\text { Agropecuaria } \\
(\mathrm{n}=487)\end{array}$ & 47 & 9.65 & 142 & 29.16 & 223 & 45.79 & 19 & 3.9 & 431 & 88.5 \\
\hline $\begin{array}{l}\text { Ciencias de la } \\
\text { Salud }(\mathrm{n}=1743)\end{array}$ & 119 & 6.83 & 305 & 17.5 & 930 & 53.36 & 96 & 5.51 & $\begin{array}{c}1 \\
450 \\
\end{array}$ & 83.2 \\
\hline $\begin{array}{c}\text { Económico- } \\
\text { Administrativa } \\
(\mathrm{n}=1309)\end{array}$ & 103 & 7.87 & 191 & 14.59 & 724 & 55.1 & 49 & 3.74 & $\begin{array}{c}1 \\
067\end{array}$ & 81.3 \\
\hline $\begin{array}{l}\text { Humanidades } \\
(\mathrm{n}=877)\end{array}$ & 80 & 9.12 & 139 & 15.85 & 467 & 53.25 & 58 & 6.61 & 744 & 84.83 \\
\hline Técnica $(\mathrm{n}=386)$ & 26 & 6.74 & 59 & 15.28 & 193 & 50 & 17 & 4.4 & 295 & 76.42 \\
\hline Total & 378 & 56 & 840 & 113.43 & 2547 & 310.13 & 240 & 29.42 & & \\
\hline
\end{tabular}

Fuente: Consumo de Drogas en Estudiantes Universitarios (CODEU, 2012).

\section{Conclusión}

El objetivo de la presente investigación fue conocer la prevalencia y el nivel de consumo de alcohol en estudiantes de la Universidad Veracruzana. Los resultados encontrados fueron: en el consumo alguna vez en la vida de $83 \%$, un porcentaje superior comparado con los resultados de la ENA 2011, donde se mostró que en la región Centro el consumo alguna vez en la vida fue de $76.9 \%$ y que en la región Sur estaban las prevalencias más bajas con $59.2 \%$.

La edad en la que comienzan a consumir alcohol la mayoría de los jóvenes es los 16 años. Resultados similares fueron encontrados por la ENA 2008 y ENA 2011, donde se indicó que el consumo iniciaba alrededor de los 17 años de edad. Por su parte, Cáceres, Salazar, Varela y Tovar (2006) obtuvieron un resultado diferente con respecto a la edad de inicio: de los 12 a los 17 años. Todos estos resultados pueden ser distintos debido a las diferentes 
culturas, contextos y políticas. Con respecto a la bebida alcohólica que más se prefiere, esta sigue siendo la cerveza, seguida del brandy, el vodka, el tequila, el ron o whisky, y por último, los cockteles (piña colada, medias de seda); todos estos datos semejantes a lo reportado por la ENA en 2011. Sobre la prevalencia, esta se entiende como la proporción de individuos de un grupo o población que presentan una característica o evento en un momento o periodo determinado. En este caso, durante el último año se encontró que la frecuencia de consumo de alcohol fue de 1 a 3 veces al mes y la cantidad ingerida de 1 a 4 copas por ocasión (20.1\%). De acuerdo al informe mundial de drogas (UNODC, 2012 p.1), el consumo de sustancias legales tuvo una mayor prevalencia: la prevalencia anual del consumo de alcohol es del $42 \%$ (teniendo en cuenta que el consumo de alcohol es legal en la mayoría de los países), cifra dos veces superior a la prevalencia anual del consumo reportado por este estudio. Con respecto al indicador uso y abuso del alcohol, la categoría consumo moderado o de riesgo bajo se refiere a la ingesta de cantidades que no causan problemas. La categoría consumo abusivo se define como el consumo que supera el volumen diario establecido en la categoría anterior, y el consumo de riesgo se refiere a un patrón de consumo de alcohol que eleva el riesgo de adquirir la adicción y de sufrir consecuencias nocivas para el consumidor, entre las que se encuentran los aspectos físicos. Al respecto se encontró que $50 \%$ de los estudiantes que participaron en este estudio presentó un nivel de consumo moderado, de ahí siguió el nivel de consumo abusivo, donde destacó el área de Artes con $21 \%$. Aunque si bien es cierto que el consumo de riesgo registró menores porcentajes, esto no deja de ser preocupante puesto que las personas que inician su consumo de bebidas alcohólicas durante la adolescencia tienen mayores probabilidades de sufrir las consecuencias del consumo excesivo de alcohol al llegar a la vida adulta, entre las cuales se encuentra el riesgo de desarrollar alcoholismo o dependencia al alcohol. Además, el consumo de bebidas alcohólicas repercute de manera importante en los accidentes vehiculares; más de la tercera parte de los conductores fallecidos por accidentes de tránsito suelen presentar una concentración de alcohol en sangre de >=0,3 gr/l (Observatorio Español sobre Drogas, Informe 2004), Finalmente, cabe señalar que el nivel de consumo de alcohol, la edad de inicio y su prevalencia pueden inducir trastornos mentales (depresión, ansiedad, inestabilidad emocional, trastorno del sueño, etcétera), no 
solo en las personas con algún trastorno ocasionado por la dependencia al alcohol, sino también en las que lo consumen de manera excesiva (ONU, 2014).

\section{Bibliografía}

Hernández S., Fernández y Baptista (2014). Metodología de la Investigación, sexta edición, editorial McGraw-Hill.

D.R@ Instituto Nacional de Psiquiatría Ramón de la Fuente Muñiz (INPRFM).

Villatoro, Moreno, Medina-Mora, Gutiérrez, Gaytán, Bretón, Sandoval, Razo, González y Amador (2008). El consumo de alcohol, tabaco y otras drogas en la UNAM: resultados de escuelas y facultades de Ciudad Universitaria, México, UNAM.

Barradas A., Martínez G., Balderrama T., Aguirre S., González S. (2009) Alcohol y tabaco en estudiantes de nivel superior. Consultada el 14 de diciembre 2014 en http://congreso.academiajournals.com/downloads/Vol\%20VI\%20Salud\%20A.pdf

Cáceres, Salazar, Varela y Tovar (2006). Consumo de drogas en jóvenes universitarios y su relación de riesgo y protección con los factores psicosociales. Pontificia Universidad Javeriana. Colombia. Consultado el 8 de enero 2013 en http://www.redalyc.org/pdf/647/64750308.pdf

De los Santos F., Barradas A., Fernández M., Sánchez, B. (2010). Edad de inicio y frecuencia en el consumo de alcohol en estudiantes de Administración y Química del ITV. Consultado 15 de julio 2914 en http://chiapas.academiajournals.com/downloads/CHIS\%20SALUD.pdf.pdf

Encuesta Nacional de Adicciones (2011). Consultado el 14 de enero de 2015 en http://www.spps.gob.mx/spps-ena-2011.html

Encuesta Nacional de Adicciones (2011). Reporte de alcohol, primera edición 2012. $\begin{array}{llllll}\text { Consultado en } & 18 & \text { de } & \text { diciembre } & 2014 & \text { en }\end{array}$ http://www.conadic.salud.gob.mx/pdfs/ENA_2011_ALCOHOL.pdf

Oficina de las Naciones Unidas contra la Droga (UNODC). Informe mundial de drogas 2012. Consultado el 14 de febrero 2015 en http://www.unodc.org/documents/dataand-analysis/WDR2012/WDR_2012_Spanish_web.pdf

Observatorio español sobre drogas. Informe 2004. Delegación del Gobierno para el Plan Nacional sobre Drogas. Ministerio de Sanidad y Consumo. Madrid, 2005 http://www.pnsd.msc.es/Categoria2/publica/pdf/oed-2004.pdf 
Organización Mundial de la Salud (2014). Alcohol. Consultado el 10 de febrero de 2015 en Alcohol en http://www.who.int/mediacentre/factsheets/fs349/es/

Organización de los Estados Americanos (2014). El Informe de drogas de la OEA OAS. Documentos oficiales; OEA/Ser.D/XXV.4.1.

Red Veracruzana de Investigación en Adicciones (REVIVA).Consultado el 13 de febrero 2015 en http://www.uv.mx/percibete/files/2012/10/DirectorioCA-REVIVA.pdf

Sistema de Vigilancia Epidemiológica de las Adicciones, SISVEA (2009). Informe 2012. $\begin{array}{lllllll}\text { Consultado en } & \text { el } & 14 & \text { de } & \text { febrero } & 2015 & \text { en }\end{array}$ http://www.epidemiologia.salud.gob.mx/doctos/infoepid/inf_sisvea/informes_sisvea _2012.pdf

Sistema de Vigilancia Epidemiológica de las Adicciones, SISVEA (2009). Informe 2012. $\begin{array}{lllllll}\text { Consultado } & \text { el } & 14 & \text { de } & \text { febrero } & 2015 & \text { en }\end{array}$ http://www.epidemiologia.salud.gob.mx/doctos/infoepid/inf_sisvea/informes_sisvea _2009.pdf

Universidad Veracruzana. Centro para el Desarrollo Humano e Integral de los Universitarios (CEnDHIU). Consultado el 14 de enero 2015 en http://www.uv.mx/cendhiu/cendhiu/quienes-somos/

UNODC. Informe Mundial sobre las Drogas 2012. Oficina de las Naciones Unidas contra la Droga y el Delito. Consultado el 12 de febrero 2015 en http://www.unodc.org/documents/data-and-analysis/WDR2012/WDR_2012_ Spanish_web.pdf 